\title{
La demografía zacatecana a comienzos del siglo XXI
}

\author{
Juan Manuel Padilla \\ Mireya A. Torres Ramírez
}

Resumen. Se aborda el estudio de la demografía del estado de Zacatecas durante el primer decenio del siglo Xxi, con el apoyo de la teoría de la transición demográfica. Se muestra, en primer término, una demografía en decrecimiento, a tono con lo que venía ocurriendo desde finales del pasado siglo. Mas se observa un súbito aumento para 2010, asociado a las bajas tasas de emigración a Estados Unidos que ha provocado la crisis económica en Estados Unidos, puesto que el crecimiento natural estatal ha seguido a la baja. En segundo lugar, se constata que vivimos un proceso de maduración y envejecimiento demográficos, con todas las implicaciones que esto conlleva. Por último, se muestra una alta dispersión poblacional y un crecimiento urbano concentrado en torno a la ciudad de Zacatecas-Guadalupe.

Palabras clave: transición demográfica, estado de Zacatecas, crecimiento, migración, envejecimiento.
Авstract. This paper is the result of a demographic study of the state of Zacate- cas, during the first decade of the century, with the support of the theory of demo- graphic transition. It shows, first of all, a de- mographicdeclining, according with what has been happening since late last century, noting, however, a sudden increase in 2010. This increase is associated with the low rates of immigration to the United Statescaused by the U.s. economic crisis, because the natu- ral increase of the state has continued to decline. In second place, it appears that, ac- cording to the last stage of the demographic transition, welive a maturation and aging of the population process, with all its implica- tions. Finally, it shows a highpopulation dispersion and urban growth concentrated around the city of Zacatecas-Guadalupe. KEYwORDs: demographic transition, Zacatecas state, growth, migration, aging.

Juan Manuel Padilla es docente investigador de la Unidad Académica de Economía de la Universidad Autónoma de Zacatecas.

Mireya A. Torres Ramírez es docente investigadora de la Unidad Académica de Economía de la Universidad Autónoma de Zacatecas. 


\section{INTRODUCCIÓN}

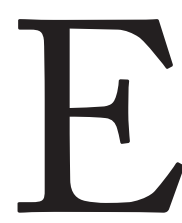
n estos primeros años del siglo xxi, México, al igual que América Latina, está experimentando un proceso de «transición demográfica». Este concepto — de obligada referencia para abordar el desarrollo en el ámbito demográfico — alude al paso de un régimen de niveles altos de fecundidad y mortalidad a niveles bajos.

Tal proceso transitorio comprende las siguientes etapas: primera, inicia el descenso de la mortalidad que, aunado a la constancia en la fecundidad, provoca un aumento significativo de la población; segunda, la fecundidad también empieza a descender, motivando una baja gradual del crecimiento, y tercera, convergen la mortalidad y la fecundidad en un nivel bajo, resultando un crecimiento bajo, nulo y, a veces, negativo. México experimentó la primera etapa entre 1920 y 1970, la segunda entre 1970 y 2000 y la tercera de 2000 en adelante (Partida, 2005). Lo anterior produce cambios en la estructura por edad de la población conocidos como maduración y envejecimiento demográfico (Miró, 2003).

La transición demográfica ocurre de manera desigual por entidad federativa. E1 Distrito Federal y Chiapas son los protagonistas extremos. Cerca del último lugar, vemos a $Z$ acatecas con valores altos de mortalidad infantil y de fecundidad. En este documento se aborda la experiencia de Zacatecas. Al respecto, postulamos que la transición demográfica se está dando de manera desigual. Por lo mismo, es importante describir las formas de esta transición, en tanto que está determinada por el nivel de bienestar social. Con este análisis se pueden ofrecer elementos que contribuyan a un diseño de políticas públicas encaminadas a mejorar dicho nivel. Es importante advertir que en otros trabajos la transición demográfica se ha estudiado como parte de los temas relacionados con la migración internacional y la pérdida poblacional derivada de este fenómeno; en este ensayo abordamos el análisis desde una perspectiva demográfica que permite dividir el estudio en tres partes: I) la 
dinámica demográfica y sus componentes; 2) la estructura por edad, y 3) la distribución territorial de la población. Por otra parte, la exposición se hace en el plano subregional.

\section{Crecimiento demográfico y SUS COMPONENTES}

E1 crecimiento demográfico expresa el movimiento conjunto de los componentes de la dinámica poblacional —mortalidad, fecundidad y migración-, de aquí la necesidad de iniciar con dicha referencia. En este apartado se describe, entonces, el ritmo de crecimiento; para ello, nos valemos de los cálculos de las tasas respectivas (a menos que se indique otra cosa).

\section{Crecimiento real}

Durante las últimas décadas, Zacatecas se ha distinguido por un ritmo de crecimiento demográfico claramente inferior al nacional, ${ }^{1}$ rasgo que persiste en 2005, cuando las tasas respectivas fueron de 0.2 y $1 \%$ anual. Esta diferencia está relacionada con la intensa emigración que caracteriza a la entidad, según la tasa de migración neta: entre 1990 y 2000 pasa de -15.2 a -18, y en 2005 pasa a -21 (véase gráfica 1). Ello sin desconocer la baja de la tasa de fecundidad que entre 1990 y 2005 pasó de 3.1 a 2.97 hijos nacidos vivos por cada mujer de 12 y más años de edad, mientras que, en los mismos años, la mortalidad pasó de 4.8 a 5.1 defunciones por cada mil (Padilla, 2008). El crecimiento natural está disminuyendo pero, como indican los datos, sus

11/ En 1990, los datos para Zacatecas y el país son, respectivamente, 1.2 y 2.04; en 1995, 0.8 y 2.06 ; en $2000,0.3$ y $1.55 \%$. 
componentes han llegado a un virtual estancamiento cuya superación implicaría avances sustanciales en materia de bienestar social, aunque no existe evidencia suficiente de que esta superación pudiese ocurrir.

\section{GRÁFICA I}

Crecimiento total, natural y social, 1970-2010.

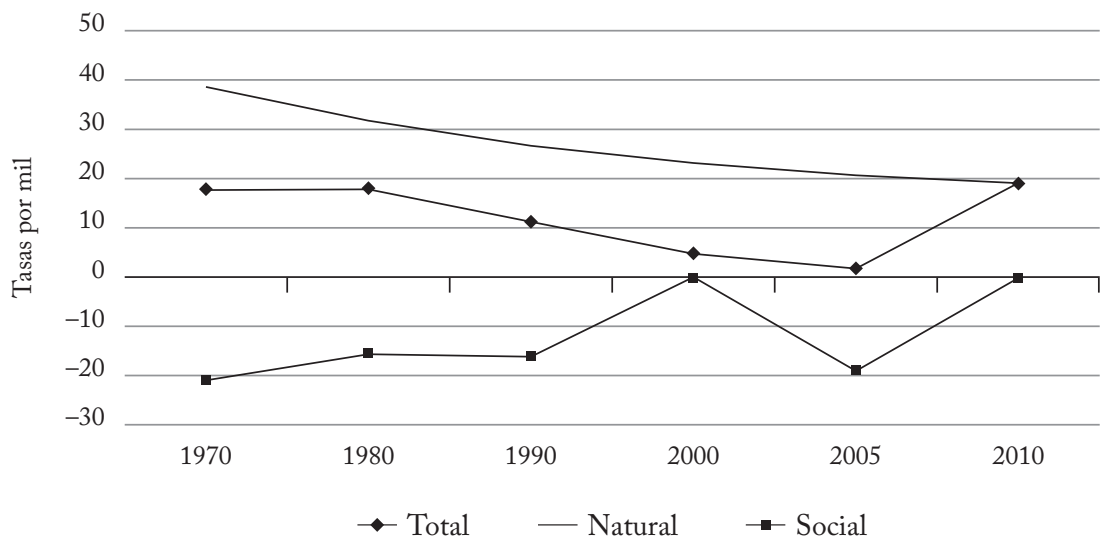

Fuente: propia con base en datos de INEGI.

Zacatecas se dirige hacia la última etapa de la transición demográfica: la población ingresó al siglo xxi inmersa en este proceso, con una tasa de crecimiento total promedio anual de 1.8 por millar para el año 2005 , inferior a la de seis por millar para 2000 y aun inferior a las tasas de años anteriores al año 2000.

La gráfica 2 ilustra el cambio demográfico de Zacatecas. Puede apreciarse la caída de la mortalidad en 1940, la cual alcanza su punto más bajo en 1990; a partir de este momento, tiende a incrementarse, aunque moderadamente, por la estructura por edad madura y «vieja». La fecundidad, por su parte, se mantiene constante, alta, y no es sino hasta 1970 que empieza su descenso. En teoría, debiera resultar un alto crecimiento demográfico, pero el modelo transitorio no se cumple en el estado debido a la intensa emigración. 
GRÁFICA 2

Transición demográfica de Zacatecas, 1940-2010.

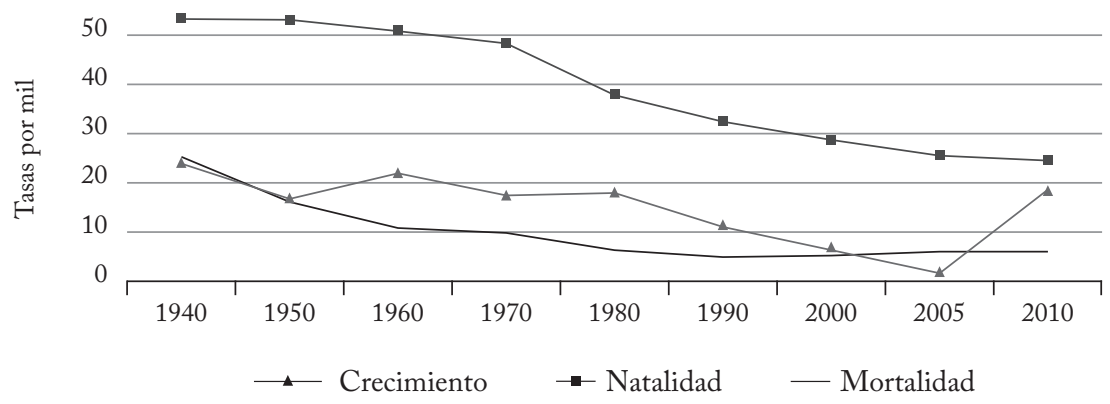

Fuente: propia con base en datos de INEGI.

Respecto de la dinámica de crecimiento, destaca la tendencia al estancamiento o decrecimiento. Esta característica o tendencia estatal se manifiesta en el plano regional, según se aprecia en el cuadro 1, donde el rasgo genérico es la tendencia a la disminución en el ritmo de crecimiento o, en su caso, aumento de la pérdida de población en las zonas de mayor emigración. La de Zacatecas aparece en los años considerados como de mayor crecimiento, seguida por Fresnillo y Pinos; todos los demás municipios tienen tasas negativas en 2000, lo cual se mantiene y profundiza en algunos casos para 2005. ${ }^{2}$ Sin embargo, es notable el hecho de que, para 2010, aparecieran, súbitamente, todas las regiones con crecimiento positivo (más adelante se explicará este fenómeno).

Buscando establecer y cuantificar la asociación entre el aumento demográfico y las condiciones de vida, procedimos a calcular los coeficientes de correlación que se presentan en el cuadro 7. Los datos indican que la correla-

${ }^{12 /}$ El esquema empleado es el del Copladez, por ser la base de las acciones gubernamentales para el desarrollo del estado y para un mayor nivel de bienestar social, permitiendo evaluar las políticas públicas y su impacto regional. 
ción entre el nivel de crecimiento demográfico y el índice de marginación es claramente negativa, es decir, que conforme aumenta la privación disminuye el crecimiento demográfico. Esto es válido para 2005 y 2010, aunque en este año se presentaron casos atípicos, como el de Jerez y Pinos; el primero ocupa el tercer sitio por su muy baja marginación y su crecimiento negativo, mientras que el segundo, de alta marginación, aumentó. ${ }^{3} \mathrm{El}$ peso estadístico de la relación es claro y significativo; en cambio, entre crecimiento demográfico y pobreza, la relación es negativa aunque no significativa en términos estadísticos.

CUADRO I

Tasas de crecimiento demográfico total regional 1990-2010 (porcentaje).

\begin{tabular}{ccccc}
\hline REGIÓn/AÑo & I990/80 & 2000/90 & 2005/00 & 2010/05 \\
\hline Zacatecas & 1.2 & 0.6 & 0.18 & 1.87 \\
Calera-Fresnillo & 1.16 & 1.82 & 0.4 & 1.63 \\
Jalpa-Juchipila & -0.22 & -0.88 & -2.1 & 0.9 \\
Jerez & -0.72 & -1.8 & -2 & 1.1 \\
Loreto-Pinos & 1.73 & 1.02 & 0.9 & 2.13 \\
Mazapil & -1.5 & -1.6 & -1.2 & 11.7 \\
Ojocaliente & 1.4 & 0.62 & 0.21 & 1.74 \\
Río Grande & 0.64 & 0.22 & -1 & 1.52 \\
Sombrerete & 0.78 & -0.5 & -1.4 & 0.6 \\
Tlaltenango & -0.58 & -1.01 & -2.8 & 1 \\
Zacatecas & 2.3 & 1.72 & 2 & 2.23 \\
\hline
\end{tabular}

Fuente: X, XI, XII y XIII Censos Generales de Población y Vivienda y II Conteo General de Población y Vivienda 2005, INEGI.

${ }^{13 /} \mathrm{El}$ índice de marginación es una medida sintética que permite diferenciar entidades federativas o municipios y localidades según el impacto global de las carencias que padece la gente. Considera cuatro dimensiones: a) falta de acceso a la educación, b) re- 


\section{CUADRO 2}

Tasas de crecimiento natural por región 1980-2005 (por millar).

\begin{tabular}{ccccc}
\hline REGIÓN/AÑo & I990 & 2000 & 2005 & 2010 \\
\hline Zacatecas & 27.2 & 24.1 & 20.4 & 18.21 \\
Calera-Fresnillo & 28.8 & 25.6 & 20.6 & 19.21 \\
Jalpa-Juchipila & 25.2 & 22.8 & 16.5 & 14.02 \\
Jerez & 24.2 & 17.1 & 12.9 & 10.48 \\
Loreto-Pinos & 30.7 & 26.8 & 23.3 & 21.59 \\
Mazapil & 26.1 & 23.5 & 18.6 & 15.75 \\
Ojocaliente & 30.9 & 24.5 & 23.4 & 22.18 \\
Río Grande & 28.8 & 23.2 & 19.7 & 18.5 \\
Sombrerete & 28.3 & 21.4 & 17.6 & 17.21 \\
Tlaltenango & 24.1 & 27.4 & 20.6 & 19.32 \\
Zacatecas & 28 & 25.1 & 20.8 & 18.78 \\
\hline
\end{tabular}

Fuente: elaboración propia con base en los XI, XII y XIII Censo General de Población y Vivienda y II Conteo general de población y vivienda 2005, INEGI.

\section{Crecimiento natural}

El despoblamiento que caracteriza al estado de Zacatecas está determinado por el descenso del crecimiento natural y el aumento de la emigración neta. Este crecimiento natural descendente es consecuencia de la política de planificación familiar implementada desde los años setenta y visible desde 1990; se aprecia en todas las regiones si comparamos 2005 y 2000 en el cuadro 3 y si

sidencia en viviendas inadecuadas, c) percepción de ingresos monetarios insuficientes, y d) residencia en localidades con menos de cinco mil habitantes. 
cotejamos con los datos de 1990. Entrados en la segunda década del siglo XxI, sólo podemos esperar que continúe dicha tendencia, aunque moderadamente.

CUADRO 3

Tasas de natalidad regional 1980-2010 (por millar).

\begin{tabular}{cccccc}
\hline REGIÓN/AÑo & I980 & I990 & 2000 & 2005 & 2010 \\
\hline Zacatecas & 37.5 & 32 & 28.7 & 25.5 & 23.69 \\
Calera-Fresnillo & 37.8 & 33.2 & 29.5 & 25.5 & 24.31 \\
Jalpa-Juchipila & 34 & 31.4 & 28.3 & 23.9 & 22.03 \\
Jerez & 32.4 & 30 & 23.7 & 21.1 & 19.54 \\
Loreto-Pinos & 45.1 & 35.3 & 30.7 & 27.7 & 26.01 \\
Mazapil & 41.9 & 29 & 26.8 & 22.9 & 21.13 \\
Ojocaliente & 43.1 & 34.9 & 28.5 & 27.7 & 27.31 \\
Río Grande & 39.3 & 33.4 & 28 & 25.4 & 24.98 \\
Sombrerete & 42 & 33 & 25.8 & 23.4 & 24.81 \\
Tlaltenango & 34.2 & 30.9 & 34.8 & 28.7 & 29.37 \\
Zacatecas & 38.5 & 32.3 & 28.8 & 24.9 & 23.00 \\
\hline
\end{tabular}

Fuente: Elaboración propia con base en los datos censales.

El dato de los eventos se tomó de los Anuarios Estadísticos de Zacatecas.

La baja del crecimiento natural es muy clara y se manifiesta en todas las regiones, sobre todo en el periodo comprendido entre 2000 y 2005, años en que prosigue el descenso con índices pequeños. Llama la atención que sea la región Jerez la de menor crecimiento natural y Ojocaliente la de mayor. Podría postularse que a mayor marginación, mayor crecimiento natural, pero este postulado no se cumple en el estado, ya que Zacatecas es la región de menor privación y no por ello es la de menor aumento natural.

$\mathrm{E} 1$ indicador del promedio de hijos nacidos vivos por mujer de $12 \mathrm{y}$ más años de edad en 2010 parece más coherente: según el Consejo Nacional de Población (Conapo), el dato para Zacatecas — municipio con menos mar- 
ginación - es de 2.5, el menor de la entidad, mientras que Mazapil, uno de los municipios con mayor marginación, tiene un promedio de 3.5, el mayor de la entidad (Conapo, 2011).

Descomponiendo el crecimiento natural, podemos constatar que la tasa de natalidad está descendiendo desde 1980, lo cual resulta claro entre 2000 y 2010 (ver cuadro 3). Si manejamos la tasa global de fecundidad, se podría afirmar que, entre 2000 y 2010, tal indicador en el país pasa de 2.8 a 2.4 en promedio de hijos nacidos vivos por mujer de 12 y más años, mientras la estatal lo hace de 3.1 a 2.44 .

CUADRO 4

Tasas de mortalidad según región, 1980-2010 (por millar).

\begin{tabular}{cccccc}
\hline REGIÓN/AÑo & I980 & I990 & 2000 & 2005 & 2010 \\
\hline Zacatecas & 5.7 & 4.8 & 4.6 & 5.1 & 5.48 \\
Calera-Fresnillo & 5.1 & 4.4 & 3.9 & 4.9 & 5.10 \\
Jalpa-Juchipila & 6.2 & 6.2 & 5.5 & 7.4 & 8.02 \\
Jerez & 5.6 & 5.8 & 6.6 & 8.2 & 9.05 \\
Loreto-Pinos & 6.8 & 4.6 & 3.9 & 4.4 & 4.41 \\
Mazapil & 4.3 & 2.9 & 3.3 & 4.3 & 5.37 \\
Ojocaliente & 5.2 & 4 & 4 & 4.3 & 5.13 \\
Río Grande & 4.9 & 4.6 & 4.8 & 5.7 & 6.48 \\
Sombrerete & 5.5 & 4.7 & 4.4 & 5.8 & 7.60 \\
Tlaltenango & 5.7 & 6.8 & 7.4 & 8.1 & 10.05 \\
Zacatecas & 5.7 & 4.3 & 3.7 & 4.1 & 4.22 \\
\hline
\end{tabular}

Fuente: cálculos propios con base en datos del INEGI.

Los datos disponibles sobre fecundidad permiten afirmar que se cumple la tesis clásica que sugiere que a mayor nivel de bienestar social menor fecundidad, y viceversa, aunque esta afirmación no pueda concluirse de manera contundente. Los municipios de Zacatecas (con 2.19 hijos nacidos vivos por 
mujer de 12 y más años) y Guadalupe (con 2.16), los de menor marginación según Conapo (2011), son los de menos fecundidad y se encuentran, de hecho, con la tasa mínima de reemplazo: 2.1. Estos municipios son, también, los de mayor nivel de participación económica femenina, de acuerdo con el Instituto Nacional de Estadística y Geografía (INEgi, 2011). Nótese, por otro lado, que el nivel de natalidad por región para 2010 es inferior al de 2000 en todos los casos. Salta a la vista la región de Sombrerete que, respecto de 2005, ha tenido un ligero crecimiento en tal indicador.

CUADRO 5

Tasas de crecimiento social por región 1980-2010 (por millar).

\begin{tabular}{cccccc}
\hline REGIÓN/AÑo & I980 & I990 & 2000 & 2005 & 2010 \\
\hline Zacatecas & -14.8 & -15.2 & -18.1 & -18.6 & 1.15 \\
Calera-Fresnillo & -15.9 & -17.2 & -7.4 & -16.6 & -2.46 \\
Jalpa-Juchipila & -24.2 & -27.4 & -31.6 & -37.5 & -5.03 \\
Jerez & -34 & -31.4 & -35.1 & -32.9 & -2.51 \\
Loreto-Pinos & -12.1 & -13.4 & -16.6 & -14.3 & 0.64 \\
Mazapil & -46.1 & -41.1 & -39.5 & -30.9 & -3.36 \\
Ojocaliente & -11.5 & -16.9 & -18.3 & -21.3 & -4.15 \\
Río Grande & -9.8 & -22.4 & -21 & -29.7 & -2.82 \\
Sombrerete & -21 & -20.5 & -26.4 & -31.6 & -11.09 \\
Tlaltenango & -28.6 & -29.9 & -37.5 & -48.6 & -7.04 \\
Zacatecas & 5.2 & -5 & -7.9 & -0.8 & 8.13 \\
\hline
\end{tabular}

Fuente: propia con base en datos del INEGI.

Desde 1940 y hasta 2000, la característica esencial de la mortalidad fue el descenso de su nivel, particularmente hasta 1980, y a partir de 2005 está creciendo moderadamente, debido a la estructura etaria madura y envejecida (ver cuadro 4). De esta forma, tenemos que entre 2000 y 2010 el nivel de mortalidad aumenta en todas las regiones, sobre todo en Jalpa, Jerez y Tlal- 
tenango, las de mayor tradición migratoria a Estados Unidos. Por su carácter selectivo, la migración involucra esencialmente a la gente joven. En este sentido, cabe subrayar que la relación entre nivel de mortalidad e intensidad migratoria internacional es positiva, con un coeficiente de correlación de 0.58 , es decir, la emigración provoca un mayor envejecimiento demográfico, lo cual se traduce en una propensión superior a morir.

Conviene agregar que la relación entre la tasa de crecimiento demográfico y la tasa de mortalidad es negativa, con un coeficiente de correlación de -0.7 , donde a mayor mortalidad menor crecimiento, y viceversa. Subrayemos también que no se da una correlación significativa al asociar la tasa de mortalidad infantil con la emigración. En efecto, como lo hemos dicho en otra parte (Padilla, 2011), la mortalidad infantil está más bien relacionada con la pobreza: en el año 2005, las regiones más pobres de la entidad — los municipios de Sombrerete, Pinos y Mazapil-, según el Consejo Nacional de Evaluación de la Política de Desarrollo Social (Coneval, 2006), son las que tienen la mayor incidencia.

\section{Crecimiento social (migración neta)}

Un rasgo común a todas las regiones en que se divide el estado es el predominio de la emigración; sólo en el caso de Zacatecas se observa una tendencia al equilibrio migratorio. Para 2010, se aprecia una disminución de la «pérdida» migratoria; incluso en Zacatecas, la migración neta es positiva, mientras que la de Sombrerete es la de mayor tasa negativa. Cabe añadir que, según datos del Programa de las Naciones Unidas para el Desarrollo (PNUd, 2008), Zacatecas registra, en 2005, el mayor índice de desarrollo humano, mientras que Sombrerete registra el menor; la relación entre tasa de crecimiento demográfico e índice de intensidad migratoria es negativa, con un grado de correlación de -0.51 , indicando que entre menor sea el número de emigrantes mayor será el crecimiento demográfico. 
En el cuadro 5 se observa el incremento de la pérdida migratoria según lo indica la tasa de crecimiento social, la cual pasa de -15.2 a -18.6 por cada mil habitantes entre 1990 y 2005. Los datos para 2010 han venido a romper con las tendencias esperadas, con 1.15 por cada mil, dando cuenta de una paralización de la migración, señal de un mercado laboral estadounidense cada vez más hermético, acompañado de una política migratoria más rígida.

En términos absolutos, tenemos que el saldo neto migratorio en 1990 era de -24,465 personas y en 2005 de -26,943; para 2010, el dato es sorpresivo: $-2,410$, según cálculos propios con base en datos del INEGI (véase cuadro 6). De este modo, el crecimiento social negativo erosiona el crecimiento natural de la población estatal. El saldo neto migratorio negativo representa, para 1990, $70 \%$ del crecimiento natural, pasando a $91.7 \%$ en 2005, mientras que para 2010 esto se revierte con un 8.8\%. En otras palabras, si en 1990 Zacatecas tenía la capacidad de retener el $30 \%$ del crecimiento natural, esa capacidad se redujo a sólo 8.3\% de tal aumento en 2005; sin embargo, ante la panorámica de la crisis actual, en 2010 se retuvo el 91.2\% (véase cuadro 6). Muchos esperábamos que en 2010 el saldo neto migratorio de la entidad superara el crecimiento natural y que, por ende, la población de 2010 fuese inferior, pero nos equivocamos.

Vemos que, en términos de intensidad, la emigración neta está incrementándose para 2005, aunque, a decir de Conapo (2006), tenderá a estabilizarse y a reducirse con el tiempo. Por lo pronto, podemos decir que, entre 1990 y 2005, el estado de Zacatecas perdió por la migración a más de 395 mil personas, cifra equivalente a más de dos veces la población del municipio de Fresnillo, el más poblado del estado.

Por municipio, veamos cómo andan las cosas para 2005: todos los municipios, salvo Guadalupe, Trancoso, Vetagrande y Villa García, tienen saldo neto migratorio negativo entre 2000 y 2005; en ellos, la emigración neta es mayor a su crecimiento natural. Entre los que tienen mayor saldo negativo durante el quinquenio 2000-2005 destacan Fresnillo con 9069 habitantes, Sombrerete con 8828 y Río Grande con 7177. De los que tienen la mayor pérdida poblacional relativa para 2005 (saldo anual/población), sobresalen 
El Plateado de Joaquín Amaro con 7\%, Momax con 6.6\% y Apozol con 6.5, según cálculos propios con datos del inegr. Todos estos municipios son de alta migración a Estados Unidos, de acuerdo con Conapo (2002).

\section{CUADRO 6}

Zacatecas: población, crecimiento natural y social 1990-2010.

\begin{tabular}{ccccc}
\hline INDICADOR & I990-I995 & I995-2000 & 2000-2005 & 2005-2010 \\
\hline $\begin{array}{c}\text { Población censada al 30 de junio } \\
\text { del primer año }\end{array}$ & 1279344 & 1331343 & 1354611 & 1366847 \\
Nacimientos durante el quinquenio & 204717 & 193690 & 180672 & 174727 \\
Defunciones durante el quinquenio & 30394 & 32039 & 33723 & 37552 \\
$\quad$ Crecimiento natural quinquenal & 174323 & 161651 & 146949 & 137175 \\
$\begin{array}{c}\text { Crecimiento natural promedio anual } \\
\text { Población censada al 30 de junio }\end{array}$ & 34865 & 32330 & 29390 & 27435 \\
$\quad$ del segundo año & 1331343 & 1354611 & 1366847 & 1491970 \\
$\quad$ Población inicial más & & & & \\
crecimiento natural (esperada) & 1453667 & 1492994 & 1501560 & 1504022 \\
Diferencia entre población censada y esperada & -122324 & -138383 & -134713 & -12052 \\
Saldo neto migratorio promedio anual & -24465 & -27677 & -26943 & -2410 \\
\hline
\end{tabular}

Fuente: elaboración propia con base en los XI, XII y XIII Censos Generales; I y II Conteos de Población y Vivienda, y los Anuarios Estadísticos de Zacatecas, ediciones 1991-2009 del INEGI.

En 2010, la pérdida migratoria disminuyó hasta el número de 2,448. En 12 de los 58 municipios se registra tasa positiva: Benito Juárez, Guadalupe, Juan Aldama, Mazapil, Pánuco y otros, aunque se trata de números muy pequeños.

\section{Migración a Estados Unidos}

A tono con la emigración a Estados Unidos, la zacatecana estuvo aumentando hasta 2005: entre 1990 y 2000 pasó de 350,276 a 513,810 personas (Conapo, 
COEPO y FNUAP, 2006) y a 579,441 en 2005 (INEgi, 2007). Si agregamos que entre 2005 y 2010 el flujo fue de 31,817 y descontamos los 10,388 retornados, según el último Censo General de Población y Vivienda, tendríamos que para el último año había 600,970 zacatecanos inmigrantes en Estados Unidos. También el flujo indocumentado está disminuyendo. Así nos encontramos en 2010.

En este contexto, puede afirmarse que la disminución de la tasa de emigración a Estados Unidos es el factor que determina el alto crecimiento demográfico de nuestro país. En efecto, los datos disponibles indican que el stock de mexicanos residentes en dicho país había llegado a su punto máximo hacia 2008: casi 12 millones, mientras que el flujo de indocumentados ha decrecido notablemente, entre otros factores, por la política migratoria disuasiva, la crisis económica y el desempleo.

Ciertamente, ha habido un aumento en las deportaciones y en los retornos voluntarios, además de que se ha inhibido la migración, sobre todo la de aquéllos que carecen de experiencia migratoria, todo lo cual ha atado a la población migrante potencial o real a sus comunidades de origen. El Censo General de Población y Vivienda encontró aquí a toda esa gente.

Por lo demás, puede señalarse que no existe cambio significativo alguno en cuanto al origen regional, mientras que cabe seguir subrayando el origen rural: $62.9 \%$ de los migrantes salen de localidades de menos de cinco mil habitantes.

En 2010, los diez municipios de mayor intensidad migratoria son Sain Alto, Apozol, García de la Cadena, Susticacán, Apulco, Momax, General Francisco R. Murguía, Nochistlán, Mezquital del Oro y Juchipila; mientras que los diez de menor intensidad son Zacatecas, El Salvador, Mazapil, Melchor Ocampo, General Enrique Estrada, Guadalupe, Cuauhtémoc, Calera, Pánuco, Luis Moya y Fresnillo. ${ }^{4}$

${ }^{14 /}$ El orden se estableció a partir del índice de intensidad migratoria, calculado por el método de los componentes principales, considerando los siguientes indicadores: a) hogares 
Si concentramos los municipios del estado por el método de conglomerados en cinco grupos, nos ubica a Sain Alto en el primero, Trinidad García de la Cadena y Apozol en el segundo, Susticacán en el cuarto, Momax en el quinto, mientras que los restantes 53 municipios se concentran en el grupo tres. Por tanto, podemos aducir que, en 2010, las zonas tradicionales de migración no han mostrado la tendencia que presentaban décadas atrás; los datos nos señalan que la migración de Guadalupe tuvo recientemente un comportamiento similar al de Tlaltenango, Juchipila, Momax, entre otros.

\section{CUADRO 7}

Coeficientes de correlación.

\begin{tabular}{cccccc}
\hline VARIABLES & MIGRACIÓN & MARGINACIÓN & POBREZA & $\begin{array}{c}\text { POBREZA } \\
\text { EXTREMA }\end{array}$ & $\begin{array}{c}\text { POBREZA } \\
\text { MODERADA }\end{array}$ \\
\hline Crecimiento & -0.188793639 & -0.335119811 & -0.027965582 & 0.02456689 & -0.087128143 \\
Migración & & 0.16048919 & 0.011897446 & 0.137426776 & -0.158739354 \\
\hline
\end{tabular}

Fuente: elaboración propia con datos del Coneval, Conapo y del Censo de 2010.

En el cuadro 7 sobresale la incidencia de ciertas variables que afectan el comportamiento demográfico y la migración en el estado. Interpretando esta incidencia, aducimos una relación negativa entre el crecimiento respecto de la marginación, migración, pobreza general y moderada. Por otro lado, en lo que respecta a la variable migración en relación con la pobreza, observamos que hay relación positiva, aunque no muy significativa. La pobreza parece no ser realmente factor de incidencia para la migración, lo cual es distinto si nos referimos a la pobreza extrema.

con migrantes 2005-2010, b) con migrantes permanentes, c) con migrantes circulares y d) con migrantes de retorno. 


\section{ESTRUCTURA POR EDAD}

Decíamos que Zacatecas está inmersa en el inicio de la última fase de la transición demográfica, cuando la fecundidad y la mortalidad han alcanzado valores bajos y en equilibrio, dando lugar a cambios sustanciales en la estructura por edad y a un bajo crecimiento demográfico. Zacatecas completará este recorrido a mediados del siglo Xxi, al igual que el resto del país.

\section{CUADRO 8}

Porcentaje regional de la población según grandes grupos de edad, 2005.

\begin{tabular}{ccccc}
\hline REGión/GRUPOS DE EDAD & TOTAL & O-I4 & I5 -64 & $65 \mathrm{Y}+$ \\
\hline Zacatecas & 100 & 32.8 & 59.4 & 7.1 \\
Calera-Fresnillo & 100 & 31.82 & 56.59 & 11.58 \\
Jalpa-Juchipila & 100 & 28.56 & 54.4 & 17.04 \\
Jerez & 100 & 25.84 & 54.27 & 19.89 \\
Loreto-Pinos & 100 & 33.69 & 57.01 & 9.3 \\
Mazapil & 100 & 28.55 & 57.23 & 14.22 \\
Ojocaliente & 100 & 33.41 & 56.96 & 9.63 \\
Río Grande & 100 & 31.02 & 56.04 & 12.93 \\
Sombrerete & 100 & 31.36 & 55.91 & 12.73 \\
Tlaltenango & 100 & 30.67 & 52.8 & 16.53 \\
Zacatecas & 100 & 30.71 & 61.56 & 7.73 \\
\hline
\end{tabular}

Fuente: elaboración propia con base en el XIII Censo General de Población y Vivienda, INEGI.

Para establecer la estructura por edad, la población se divide en tres grandes grupos. En 1970, la población menor de 15 años ascendía a 50.5\% de la población total, la de entre 15 y 59 años a $43.3 \%$ y la de 60 y más años a $6.2 \%$. En 2005, estos datos representan, respectivamente, 32.4, 57.8 y $9.8 \%$ según el INEGI (véase cuadro 8). Nótese que el grupo de menores de 15 años 
de edad es inferior al de 15-64 en todos los municipios, mientras que en 1970 era al contrario. Vivimos, pues, un proceso de maduración demográfica, más que de envejecimiento, lo cual implica nuevos y complejos desafíos, tanto para la política pública como para la sociedad en general.

\section{GRÁFICA 3}

Pirámide de población de Zacatecas, 1970-2010.

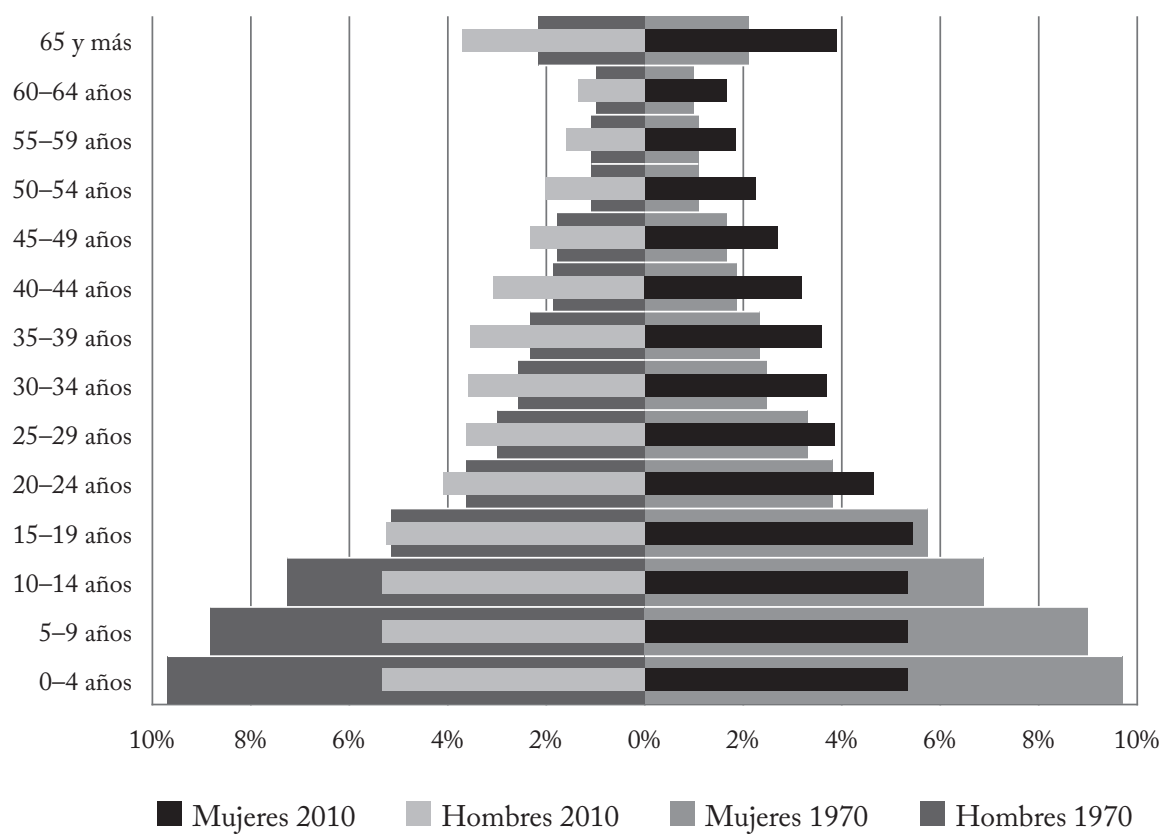

Fuente: elaboración propia en base a datos del IX y XIII Censos Generales de Población y Vivienda, INEgI.

Tal proceso está ocurriendo de manera desigual por municipio. Para 2010, Zacatecas es el de mayor porcentaje de población de entre 15 y 64 años de edad, lo cual refleja la atracción que ejercen Guadalupe y Zacatecas sobre la gente de otros municipios, porque concentran el principal cambio en la economía estatal durante los últimos años: su terciarización. En cambio, las regiones históricas de migración internacional son las de mayor nivel relativo 
de población de 65 y más años, pues quienes emigran son, esencialmente, jóvenes; en este caso, la migración contribuye al despoblamiento y al envejecimiento, mientras que en el primer caso la rejuvenece.

\section{GRÁFICA 4}

Población de sesenta y más años, 1970-2010.

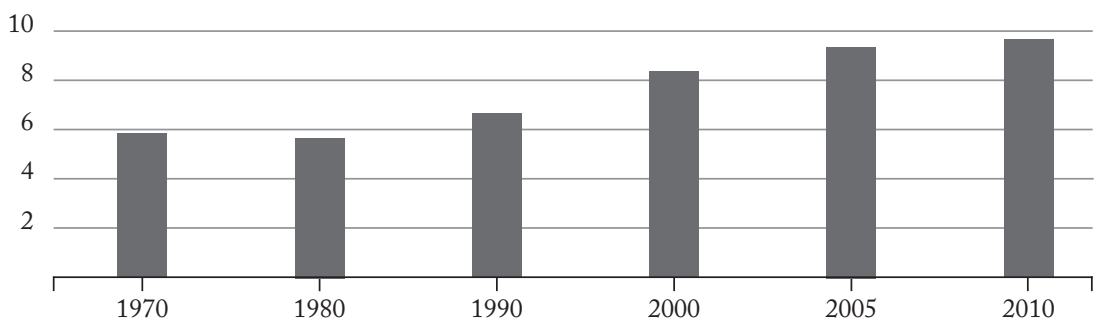

Fuente: propia con base en datos censales.

El porcentaje de población de 65 ó 60 años y más se toma frecuentemente como indicador del envejecimiento, pero la extensión de la edad mediana de la población suele ser mejor. Tal edad pasó de 21 a 25 años entre 2000 y 2005 en el estado, mientras que en el país pasaba de 22 a 26. De cualquier modo, es ilustrativo tal porcentaje; de cara al futuro, podemos decir que se profundizarán los cambios mencionados (véase gráfica 3). Primero, el grupo de población menor de 15 años continuará a la baja hasta 2030; segundo, el grupo de 15 a 59 años crecerá hasta 2015 para luego disminuir; finalmente, el grupo de 60 y más crecerá consistentemente durante el tiempo proyectado, empatando hacia 2030 el nivel de menores de 15 años de edad (véase gráfica 4).

Lo anterior implica enormes retos en los planos económico, social y político. En lo económico, será inviable seguir sosteniendo la seguridad social, por ser pocos los que cotizan a ésta y por ser pequeña la aportación. Si los problemas de empleo siguen persistiendo, será alto el número de personas de edad avanzada sin alguna seguridad social. Se debe fortalecer la política 
de apoyos a ese segmento poblacional para evitar la asociación entre edad avanzada y pobreza.

\section{CUADRO 9}

Población por grandes grupos de edad 2005-2030 (porcentaje).

\begin{tabular}{cccc}
\hline AÑO & \multicolumn{3}{c}{ GRUPOS DE EDAD } \\
\hline 2005 & $0-14$ & $15-59$ & $60 \mathrm{y}+$ \\
2010 & 33.3 & 57.7 & 9 \\
2015 & 29.6 & 60.4 & 10 \\
2020 & 26.1 & 62.4 & 11.5 \\
2025 & 23.7 & 62.7 & 13.6 \\
2030 & 22 & 61.5 & 16.4 \\
\hline
\end{tabular}

Fuente: proyecciones del conapo (2011).

\section{DISTRIBUCIÓN TERRITORIAL DE LA POBLACIÓN Y PROCESO DE URBANIZACIÓN}

El patrón de poblamiento de Zacatecas, igual que el del país, muestra dos características: por un lado, una alta dispersión en cientos de localidades y, por el otro, su concentración en pocos centros urbanos. Empecemos por descomponer la población según tipo de residencia.

La población rural, residente de localidades menores de cinco mil habitantes, ha crecido a una tasa menor a la estatal, según se aprecia en la gráfica 2; decrece en los últimos años, incluso, para crecer a $0.9 \%$ promedio anual entre 2005 y 2010. Con todo, la población rural sigue siendo predominante en la población total: en 2010, 48.5\% es rural, $11.3 \%$ es mixta y $40.2 \%$, urbana. Lo anterior no es ajeno a la debilidad de nuestra economía regional dentro de la nacional, 0.8\% en 2008 según datos del inEGi (2010). 
CUADRO IO

Localidades según tamaño y población, 2010.

\begin{tabular}{ccccc}
\hline TAMAÑO & LOCALIDADES & POBLACión & RELATiVOS & \% ACUM \\
\hline $1-249$ & 3953 & 148121 & 9.94 & 9.94 \\
$250-499$ & 306 & 109009 & 7.31 & 17.25 \\
$500-999$ & 212 & 148519 & 9.96 & 27.21 \\
$1000-2499$ & 134 & 198010 & 13.28 & 40.49 \\
$2500-4999$ & 33 & 114214 & 7.66 & 48.15 \\
$5000-9999$ & 17 & 106916 & 7.17 & 55.32 \\
$10000-14999$ & 5 & 66959 & 4.49 & 59.81 \\
$15000-29999$ & 6 & 115202 & 7.73 & 67.54 \\
$30000-49999$ & 3 & 109725 & 7.36 & 74.9 \\
Más de 100000 & 3 & 374578 & 25.13 & 100 \\
Suma & 4672 & 1490668 & 100 & 100 \\
\hline
\end{tabular}

Fuente: elaboración propia con base en el XIII Censo General de Población y Vivienda, INEGI.

La población mixta, residente de localidades de entre cinco mil y 14,999 habitantes, parece tener una contribución relativa constante. Es un tanto irregular por la reclasificación de localidades mixtas a urbanas o de rurales a mixtas. $7.7 \%$ de la población estatal reside en localidades de entre cinco mil y 9999 personas y que, en el estado, suman 16, entre otras: Villa de Cos, Juchipila, Pinos, Villa García, General Francisco R. Murguía y Tacoaleche; mientras que 4.5\% reside en las que oscilan entre 10 mil y 14,999 habitantes: Miguel Auza, Villanueva, Trancoso, Jalpa y Valparaíso.

El crecimiento demográfico que vive Zacatecas es, fundamentalmente, urbano, o sea, el que se da en las localidades de 15 mil y más habitantes; éste fue del orden de 3.65\% promedio anual en 2010, claramente superior al estatal. En total, suman doce localidades; Zacatecas, Guadalupe y Fresnillo tienen más de 100 mil habitantes, y las demás tienen menos de 50 mil: tres 
entre 30 mil y 49,999, tres entre 20 mil y 29,999 y tres entre 15 mil y 19,999. En estas localidades vive $40.15 \%$ de la población estatal.

\section{GRÁFICA 5}

Poblacion total, rural, urbana y mixta, 1970-2010.

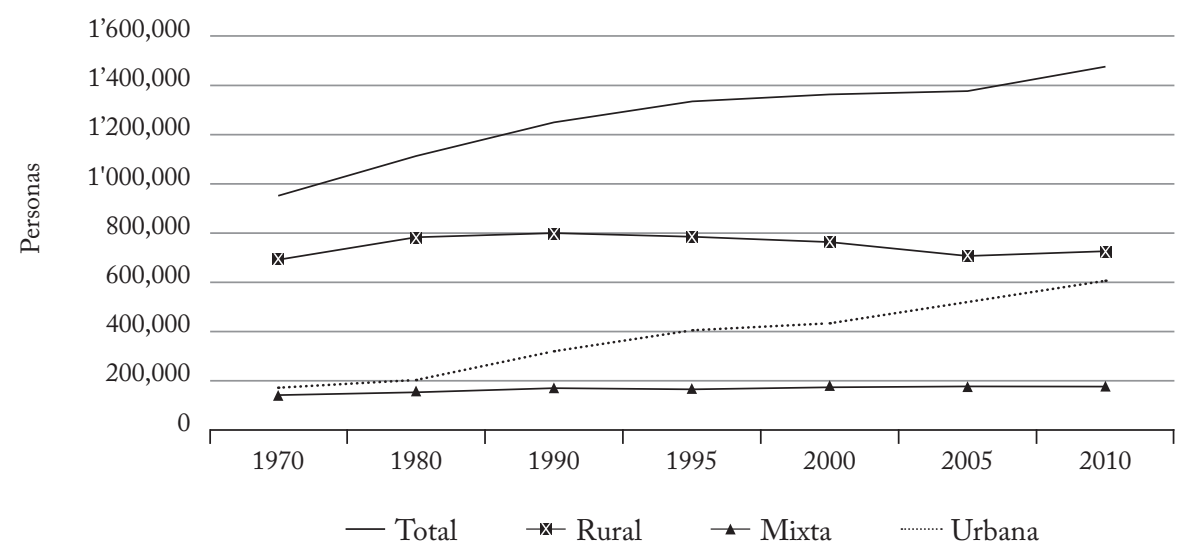

Fuente: Censo general de Población y Vivienda, INEGI.

Los datos disponibles permiten afirmar que el proceso de urbanización es débil en la mayoría de las regiones, con localidades urbanas pequeñas, que apenas crecen; vemos esto en el noroeste y suroeste, Nochistlán y Sombrerete, por ejemplo. Vivimos un proceso de urbanización polarizado, fundamentalmente en la zona metropolitana Zacatecas-Guadalupe, lugar que concentra la terciarización que registra la economía, aportando 32\% del producto estatal en 2004; en 2010, 20\% de la población estatal vivía en estas localidades.

El municipio de Guadalupe registra el mayor proceso de urbanización, lo cual se relaciona con la conurbación de las localidades de Guadalupe y Zacatecas, constituyéndose de esta forma en la primera zona metropolitana estatal. Realmente, el alto crecimiento demográfico que caracteriza al municipio es el experimentado por la localidad de Guadalupe, cuya población pasa, entre 1980 y 2010, de 25,395 a 124,623 habitantes, esto es, casi se mul- 
tiplica por cinco veces. Si esta localidad continúa creciendo con la intensidad que lo hizo entre 2005 y 2010, 5\% promedio anual, para 2024 se tendría que construir otro Guadalupe con todos sus servicios: viviendas (más de $31 \mathrm{mil}$ ), empleos (alrededor de 33 mil), transporte urbano, entre otras, para hacer frente a una población de 250 mil habitantes, lo doble que en 2010. ¿Qué tan deseable sería un proceso de urbanización marcadamente polarizado en esta localidad? ¿Y los demás sectores y regiones?

CUADRO I I

Localidades urbanas 2000-2010, habitantes y aumento.

\begin{tabular}{cccccc}
\hline LOCALIDAD/AÑo & 2000 & 2005 & 2010 & r2005/2000 & r2010/2005 \\
\hline Zacatecas & 113947 & 122889 & 129011 & 1.39 & 1.05 \\
Fresnillo & 97043 & 110892 & 120944 & 2.52 & 1.89 \\
Jerez & 37558 & 38624 & 43064 & 0.5 & 2.37 \\
Guadalupe & 78879 & 99572 & 124623 & 4.64 & 4.95 \\
Rio Grande & 29214 & 29309 & 32944 & 0.06 & 2.55 \\
Calera & 25709 & 29626 & 32721 & 2.69 & 2.16 \\
Sombrerete & 18668 & 19353 & 21702 & 0.65 & 2.5 \\
Loreto & 19634 & 22085 & 24260 & 2.21 & 2.04 \\
Nochistlán & 15499 & 15322 & 16562 & -0.2 & 1.69 \\
Ojocaliente & 18150 & 18940 & 20851 & 0.77 & 2.09 \\
Juan Aldama & - & - & 15431 & - & - \\
Tlaltenango & - & - & 16396 & - & - \\
Suma & 454301 & 506612 & 598509 & 2.03 & 3.65 \\
\hline
\end{tabular}

$r=$ tasa de crecimiento promedio anual

Fuente: elaboración propia con datos del XII y XIII Censo General de Población y Vivienda y II Conteo General de Población y Vivienda, INEGI.

Queda claro que el proceso de urbanización se concentra en el centro de la entidad. Sería deseable un proceso de urbanización menos inequitativo, territorialmente hablando. Sabemos, por otra parte, que el desigual cambio 
económico que vivimos se ha acompañado de una dispareja distribución poblacional por el territorio, destacándose tres tendencias: primera, su creciente concentración en la región de Zacatecas, particularmente en los municipios de Zacatecas y Guadalupe; segunda, un crecimiento moderado en el centro norte y el sur: Calera-Fresnillo y Loreto-Pinos; tercera y última, un decrecimiento en el occidente y en el norte. No es un hecho nuevo, aunque sí es notorio que para 2005 todos los municipios de las regiones de Jalpa, Jerez, Tlaltenango, Sombrerete y Río Grande registran despoblamiento, además de otros de las demás regiones. La distribución de la población por el territorio se corresponde más o menos con la del crecimiento económico: para 2005, Calera-Fresnillo, a título de ejemplo, concentra $22 \%$ de la población y aporta $21.2 \%$ del producto estatal.

\section{CUADRO I 2}

Porcentaje regional de la población de Zacatecas, 1970-2010.

\begin{tabular}{cccc}
\hline REGIón/AÑo & I970 & I990 & 2ого \\
\hline Zacatecas & 100 & 100 & 100 \\
Calera-Fresnillo & 19.3 & 20.2 & 21.9 \\
Jalpa-Juchipila & 11.8 & 9.1 & 6.9 \\
Jerez & 12.2 & 9.4 & 7.1 \\
Loreto-Pinos & 10.2 & 11.4 & 12.3 \\
Mazapil & 5.6 & 3.3 & 2.4 \\
Ojocaliente & 5.7 & 6.5 & 7.5 \\
Río Grande & 9.6 & 10.2 & 9.1 \\
Sombrerete & 8.4 & 8.2 & 6.6 \\
Tlaltenango & 6.4 & 4.86 & 3.7 \\
Zacatecas & 11.3 & 17.1 & 22.5 \\
\hline
\end{tabular}

Fuente: elaboración propia con datos del IX, XI y XIII Censos Generales de Población y Vivienda, INEGI. 
El crecimiento demográfico diferenciado se refleja en variaciones en la aportación relativa por municipio o región respecto de la población estatal. En el primer plano, sobresalen Fresnillo con 14.3\%, Guadalupe con 10.7\%, Zacatecas con 9.3\%, Pinos con 4.7\% y Río Grande con 4.2\%. Cotejando los datos de 2005, destaca Guadalupe que «arrebata» a Zacatecas el segundo lugar. Por región, la de Fresnillo le cede en 2010 el primer sitio a la de Zacatecas. Otras que vieron disminuida su contribución son Jalpa, Jerez, Ojocaliente, Río Grande, Sombrerete y Tlaltenango, mientras dos la mantuvieron, Pinos y Mazapil.

En materia de cómo se distribuye la población por el territorio se sigue apreciando una alta dispersión, además de un proceso de concentración urbana. Lo primero continuará complicando de manera extraordinaria el acceso a los servicios, mientras lo segundo seguirá mostrando la problemática propia de la urbanización.

\section{Conclusiones}

Los datos del Conteo del año 2005 permiten confirmar algunas características y tendencias de la población zacatecana de finales del siglo xx: un nivel menor de crecimiento que tiende al estancamiento; un aumento del despoblamiento; un decrecimiento del grupo de menores de 15 años de edad y un aumento en los demás, lo cual muestra una estructura etaria más madura y envejecida; crecimiento de la población urbana, esencialmente en torno a las ciudades de Zacatecas y Fresnillo, y una disminución de la rural; una creciente concentración en la región de Zacatecas, especialmente en los municipios de Zacatecas y Guadalupe, lo cual refleja la desigualdad del cambio económico que vive la entidad.

Los determinantes del despoblamiento son un decreciente aumento natural y un incremento de la emigración neta, particularmente la dirigida a Estados Unidos. Parece que el stock de zacatecanos residentes en dicho país había llegado a su punto máximo hacia 2008 y que el flujo migratorio 
indocumentado había decrecido notablemente por la política disuasiva, la crisis económica y el desempleo, entre otros factores. Una menor tasa de emigración externa es lo que explica la alta tasa de crecimiento demográfico de 2010, además de la activación minera en algún municipio. Los datos disponibles sobre el crecimiento natural no validan, al parecer, el aumento de la fecundidad como factor relevante del crecimiento aludido.

Es posible que el rol que desempeñaba la migración como «válvula de escape» ya no siga cumpliéndose; en ese caso, habría que preguntarnos si el estado está preparado para asimilar a los migrantes. Antes, los jóvenes encontraban en Estados Unidos lo que la economía mexicana no les ofrecía, pero ese país ha cancelado esta opción.

Como resultado de los cambios en la fecundidad, la mortalidad y la migración, vivimos el inicio de la última fase de la transición demográfica, o sea, un proceso de maduración y envejecimiento demográfico. Las condiciones de vida de los adultos mayores deben mejorar con programas de apoyo universal. El envejecimiento demográfico es inevitable, pero sí podemos evitar la pobreza o, al menos, moderarla.

Seguimos observando una alta dispersión de la población por el territorio. Podrían implementarse políticas de focalización en comunidades dispersas; éstas deberían incluir servicios locales concentrados como agua, luz, salud y vivienda, en busca de estimular economías de escala en la concentración de servicios públicos que favorezcan una mejor calidad de vida, complementando la política de combate a la pobreza. También vivimos un proceso de urbanización polarizado. Sería deseable un sistema de ciudades más equitativo, territorialmente hablando. Además de programas de combate a la pobreza urbana.

Queremos decir, finalmente, que reivindicamos la utilidad de la transición demográfica para el estudio del cambio poblacional. 


\section{ReFERENCIAS}

Conapo (2002), Indice de Intensidad Migratoria México-Estados Unidos, 2000, Aguascalientes, Conapo.

(2006), Índices de marginación 2005, Aguascalientes, Conapo.

(2011), Indice de marginación por entidad federativa y municipal 2010, Aguascalientes, Conapo.

Conapo, coepo y fnuap (2006), Sistema de Información de Migración Internacional del Estado de Zacatecas, Zacatecas, s.e.

INEGI (2006), II Conteo general de población y vivienda 2005, Aguascalientes, INEGI.

(2007), Encuesta Nacional de la Dinámica Demográfica (ENADID) 2010, Aguascalientes, INEGI.

(2011), Censo general de población y vivienda 2010, Aguascalientes, INEGI.

Padilla, Juan Manuel (2008), Cambio demográfico y desigualdad social en Zacatecas, Aguascalientes, Poder Judicial del Estado de Zacatecas.

(1 de noviembre de 2011), «De muertos en Zacatecas», La jornada Zacatecas.

PNUD (2008), Índice de desarrollo municipal en México, 2000-2005, México.

Partida Bush, Virgilio (2005), «Transición demográfica y el proceso de envejecimiento en México», Papeles de Población, vol. 11, núm. 45.

VAldés, Luz María (2000), Población, reto del tercer milenio. Curso interactivo introductorio a la demografía, México, Universidad Nacional Autónoma de México, Porrúa. 